\title{
Liver and Tumor Segmentation Techniques for CT Abdominal Images
}

\author{
Hema N, Laxmidevi Noolvi, M V Sudhamani
}

\begin{abstract}
Image segmentation is one of the important step in digital image processing where the images are partitioned into different segments based on several properties like brightness, contrast, intensity and texture. Image processing includes several steps among which image segmentation is the difficult task. Accurate segmentation is the fundamental step in digital image processing. Segmentation can be performed manually, but as it is a tedious task, automatic segmentation techniques which gives more accuracy has to be found. Many recent segmentation techniques for liver image segmentation are discussed here. Some of the techniques to segment liver from CT scanned abdominal image and to segment tumor from the liver are discussed. The main objective is to highlight various techniques which can aid in developing a novel segmentation technique.
\end{abstract}

Index terms - Abdominal image, Liver, Enhancement, Segmentation, CT scan images.

\section{INTRODUCTION}

Medical imaging is the approach used to create visual representations of the internal parts of the body for diagnosis and treatment. The proposed Computer Aided Diagnosis (CAD) system includes various stages as shown in below Figure 1. Liver segmentation plays responsible role in understanding the functions of liver which in turn helps in liver disease diagnosis. In this paper more importance is given to Computed Tomography Abdominal Images. Segmentation of liver from a abdominal CT scanned image has an apparent role because it aids the radiologist to assist doctors for diagnosis. The main purpose of segmentation is to modify the representation of an input image into different form which will be easier for analysis.

The objects and boundaries of an image can be located using segmentation techniques. Segmentation process can be performed either using automatic method or Semi-automatic method.

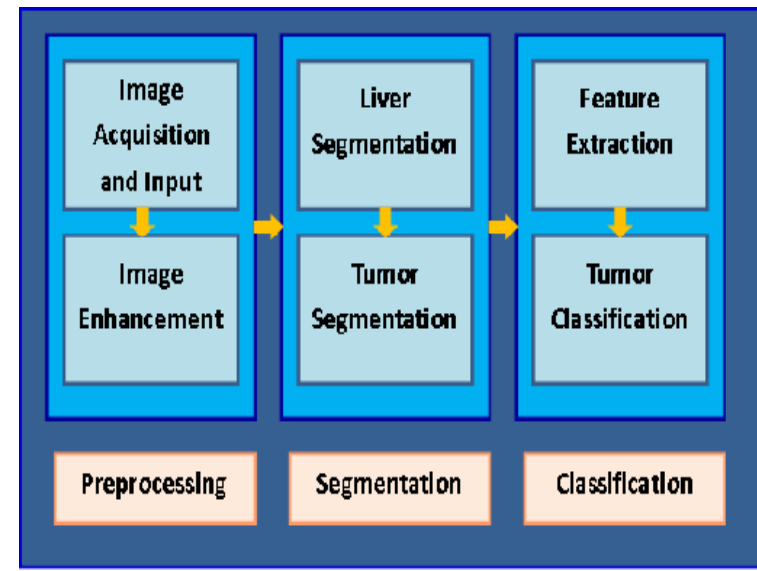

Figure 1 Proposed CAD system

Automatic segmentation is the process where boundaries of an object are assigned by a program automatically. Semi-automatic segmentation is the methodology which includes human intervention for selecting, checking and editing segment boundaries. The Abdominal CT scanned image is shown in below Figure 2.

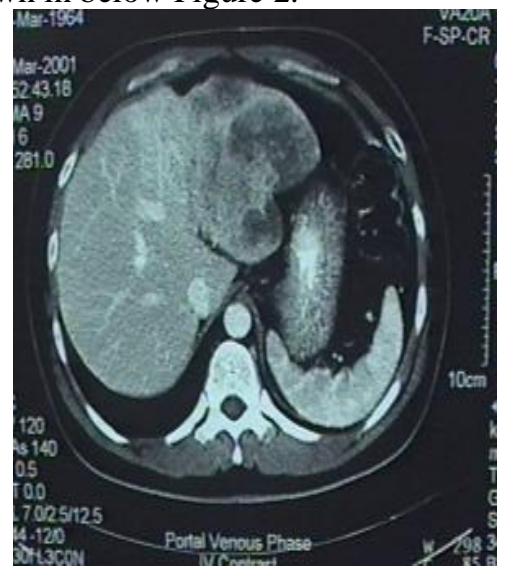

Figure 2 Abdominal CT scanned image

\section{DISCUSSION OF TECHNIQUES}

In this paper, various methods of segmentation that are applied in medical image analysis are discussed. The segmentation methods are classified into different categories such as, 1) Thresholding methods

2) Region based methods 3) Clustering and classification methods 4) Model-based methods.

\section{Threshold based methods}

A simple method used for segmentation is Thresholding. This method is used as the first step in most of the segmentation process. The method can be applied when the contrast between the foreground and the background is more.

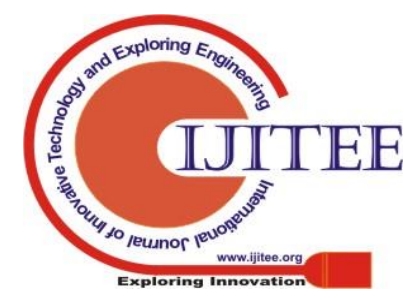




\section{Liver and Tumor Segmentation Techniques for CT Abdominal Images}

Thresholding technique results in a binary image. This simple method replaces every pixel in an image with black pixel when the image intensity $I_{i, j}$ is below than some set constant value $\mathrm{T}$. If the intensity of an image is greater than $\mathrm{T}$ replaces with white pixel. Thresholding methods can be mainly classified into two categories as discussed below.

a) Global Thresholding: In global thresholding technique intensity of every pixel of an image is compared with one threshold value. The pixel intensity values are greater than the set threshold values are set to one and remaining is set to zero. This results in a binary image. The choice of optimum threshold value is very important. One of the basic methods to automatically find the valid value for threshold is Otsu's thresholding method. This method generates the histogram and the valid threshold value is selected to divide the image into two classes. The optimal threshold value is selected in such a way that the difference between the two classes is more.

b) Local Thresholding: Local thresholding is also known as adaptive thresholding method. This method makes use of threshold values that are locally available instead of using a one threshold value for the complete image. Local threshold values can be estimated by computing mean of intensity values that are present as pixels in the neighborhood for every point in an image.

\section{Region based segmentation methods}

A group of pixels which are connected based on similar properties form a region of an object or it can also be a part in an object. Regions are formed by using methods related to regions by combining pixels available in the neighborhood. The region is computed by considering the defined similarity values between the pixels. Using this type of methods images can be divided into different regions where there is no overlap between them and the original image is obtained by taking the union of these regions. One of the basic region based segmentation method is the region growing algorithm.

a) Region Growing: The basic and simple region based method used for the segmentation purpose is Region Growing (RG). Here one of the seed point has to be initialized automatically. The selected seed point is located in the area of interest which has to be segmented. The seed point is compared with the neighbor pixel based on similarity criteria one after one. If any of the neighbor satisfies this condition then that is added to the region. The similarity criteria used is the intensity value of the image.

\section{Clustering and Classification methods}

Classification technique is used to segment the objects into different classes and these are known as supervised learning techniques. Here the target class is already known and then training data is used to train the classifier in such a way that new test data is classified accordingly. Some of the classification methods are K-Nearest Neighbor Classifier, Support Vector Machine classifier and Naïve Bayes classifier, and so on. The set of objects which are more similar are grouped into same cluster where cluster is a collection of similar entities. This process is known as clustering. Clustering techniques are known as unsupervised learning. The similarity measure is found by estimating the measure of distance by using Euclidean distance method. Some of the commonly and frequently used methods are K-means clustering, Fuzzy C-means (FCM) clustering, and so on.

\section{Deformable model segmentation methods}

One of the challenging tasks is to segment 3-Dimensional images. Some of the model based segmentation approach such as parametric and geometric deformable models are used. Segmentation of images which has anatomic structures is done by using Deformable models. This is carried out by constructing connected and continuous model where information about the location, size, orientation, and shape of these structures are considered in prior.

The variations of biological structures over time and across different individuals can be adjusted using deformable models. The two classes of deformable models are Parametric and Geometric.

a) Parametric Deformable Models: The other name for Parametric Deformable Models (PDM) is called as snakes. Snakes are the curves which lie within the domain of an image. These curves are made to move by applying forces internally and externally. The contour in PDMs are the parametric curve $\left(\mathrm{E}_{\mathrm{snake}}\right)$ which is estimated by taking the summation of the energy terms $\mathrm{E}_{\text {constraint }}, \mathrm{E}_{\text {extrenal, and }} \mathrm{E}_{\text {internal, }}$, where $E_{\text {constraint }}$ is the energy component which is defined based on the curve constraint. $E_{\text {extrenal, and }} E_{\text {internal }}$ which are used in summing are referred as the components of energy resulted by applying external force and internal force.

The goal of defining the forces in an intelligent manner is very important because final contour position must have minimum energy. This leads to the problem of minimization of energy. When the object boundaries are very close to one another, the position selected initially from the model must be placed closer to the required boundary. This selection helps to overcome the problem of converging into wrong boundaries.

b) Geometric Deformable Models: It is very difficult to change the curve topology in PDMs as it starts evolving. When there is change in shape re-parameterization of the curve has to be performed. The solution for this problem is to use Geometric Deformable Models which is also known as Level Sets (LS). LS is evolved to fit and also to track the objects which is of our interest. This is achieved by making modification to the embedding function where as curve function is modified in PDMs. These two methods makes an improvement in initializing parametric active contours and then initial contours are placed symmetrically. It is not easy to achieve accurate segmentation results in segmentation as objects to be segmented may have irregular shapes.

\section{RESULTS AND OBSERVATIONS}

In [1], a novel liver segmentation method for Computerized Tomography (CT) images which includes separation of intensity values, Region Growing and morphologic operations are proposed. First it is very important to extract liver from CT scanned abdominal images. Histogram analysis is used to find the gray levels of the liver object.

The separation based on intensity is carried to improve the differences that exist between the liver intensity values and the intensity of other tissues

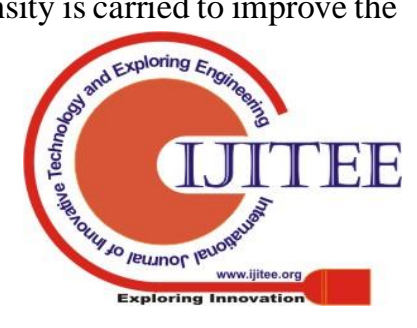


which are surrounding liver. Liver is segmented by applying region growing algorithm. To improve the segmentation result some of the morphological operations are applied. This framework results in reliable and robust form of segmentation of the liver image.

In [2], a graph cuts method is modified automatically and features are detected which helps in segmentation of liver accurately and fastly is proposed here. Intensity method along with the information of the position is used to determine the slice and seed points initially in the graph cut method. Anatomical information of vessel is used to detect the features and the adjacent vena cava which has similar intensities is eliminated. The experiments are carried on various datasets like, 20 3Dircadb, 20 Sliver07 datasets and clinical datasets. The average volumetric overlap error is $5.3 \%$, volume difference achieved is $0.6 \%$, symmetric surface distance is $1.0 \mathrm{~mm}$ and volume processing time is $17.8 \mathrm{~s}$ for the Sliver07 dataset and 8.6\%, 0.7\%, $1.6 \mathrm{~mm}, 12.7$ $\mathrm{s}$ for the 3 Dircadb dataset respectively. This method helps to extract the liver more accurately from the low contrast and difficult backgrounds. The method discussed here is fully automatic and aids in segmenting the liver more accurately.

In [3], to segment liver tumor automatically Multiple Kernel Fully Convolution Networks (MK-FCN) is discussed. MK-FCN segments the liver tumor which is present in the contrast-enhanced CT images by considering various characteristics of quality of scan and tumor in various phases. The multiple convolution kernels are provided by MK-FCN which convolutes the CT images in various enrichment phases and feature combination in the network are realized. The proposed work was performed on two databases such as 3Dircadb and JDRD. By applying FCN for the 3Dircadb dataset, the obtained mean ratios of MSD is $7.1 \mathrm{~mm}$ and RMSD is $2.9 \mathrm{~mm}, \mathrm{RVD}$ is $5.8 \%$, VOE is $14.0 \%$, ASD is $2.0 \%$, For JDRD dataset by applying MK-FCN, the estimated mean ratios of RVD, VOE, ASD, RMSD and MSD are 5.2\%, 10.1\%, 1.7\%, 2.1 mm and 6.1mm respectively.

In [4], the techniques to detect and segment liver tumors which are present in noised computed tomography images is proposed. Fuzzy C-Means clustering (FCM) algorithm is used to segment the noise free images. Next, Distance Regularized Level Set Evolution (DRLSE) is applied to detect and extract the tumor boundaries. FCM is sensitive to noise and this results in over segmentation. To overcome this problem median filter is used on noisy images. After performing FCM segmentation, the segmented image is given to DRLSE method from which tumors are extracted from the liver.

In [5], the segmentation of liver by three-dimensional mesh deformation models and liver tumor segmentation by SVM framework are proposed. This technique was tested on 20 datasets. For each CT image in the dataset, the medical experts re manually marked the Liver and its tumor, it is considered as Reference Standards (RS). The results of proposed method were compared with RS using the Jaccard index based on voxel matching. For human liver and tumor, an average Jaccard similarity index value is $0.93+-0.05$ and $0.75+-0.05$ respectively was achieved.

In [6], Proposed the region growing segmentation of liver using mean intensity as seed points. Next, edge detection is performed on the liver in order to get the edge points, mean of the edge pixels serves as the Region of Interests (ROIs) centers. The texture features like Gray level co-occurrence matrix are extracted for each ROIs. The SVM classifier is used to train and classify, wherein extracted feature vector of labeled tumor regions are selected as positive examples and features of selected liver regions are considered as negative examples. The average prediction accuracy of treatments is measured up to $82.3 \%$.

In [7], the CAD system proposed consists of enhancement of image, segmentation of liver, extraction of feature and the characterization of $\mathrm{HCC}$ is carried by using classifiers. Contrast Limited Adaptive Histogram Equalization (CLAHE) technique is applied for pre-processing, Morphological operations to segment the liver, Discrete Wavelet Transform (DWT) is used for feature extraction and Support Vector Machine (SVM) is applied to classify tumors for HCC diagnosis. The proposed system results in tumor classification accuracy of $88.46 \%$.

In [8], to obtain the gradient image morphological operation along with gradient transformation was carried on the input image. Then level set method is used to segment the image. The evaluation parameter used here is the similarity coefficient. The similarity result obtained after applying this method is more than $95 \%$.

In [9], Median filtering is used for preprocessing an image. Simple Linear Iterative Clustering technique is adopted to segment the tumor from the segmented liver image. Gray Level Co-occurance Matrix (GLCM) and histogram features are used for feature extraction. Hamming Distance is used to validate tumor. This method has achieved the average accuracy of $91.97 \%$.

In [10], a Single-Block Linear Detection Algorithm (SBLDA) algorithm is used for extracting liver from the CT images. The edge of the liver is extracted by applying morphological operations by using confidence matrix which is calculated by using SBLDA technique. The accuracy achieved is higher than $96 \%$ when the threshold value $T$ was ranging between 0.82 and 1 . The outliers present were removed by applying morphological filtering in which the structuring element disk was considered with a radius of two pixels. Median filtering was used to reconnect the disconnected structures of the liver edge. The average sensitivity achieved for liver cancer was $96.59 \%$, accuracy was $98.65 \%$, and the obtained specificity was $99.03 \%$.

In [11], the proposed system is used for automatic segmentation of lesions from 3 dimensional liver CT images. A mask is generated to guess the segmentation area by using initial facts of the location and shape. Then using thresholding and morphological processing techniques liver is segmented. Gaussian filter is applied to eradicate noise and safeguard the edges to detect the lesions. The experiments conducted using 31 cases has obtained $92.68 \%$ accuracy

In [12], Fuzzy C-Means Clustering (FCM) and Grey Wolf Optimization (GWO) together forms an hybrid approach which is carried out for automatically segmenting the liver. Fast fuzzy c-means clustering (FFCM) is applied for feature extraction and for extracting candidate lesion.

Next, the classification of the lesions is done using support vector machine technique. Now, the liver image which is

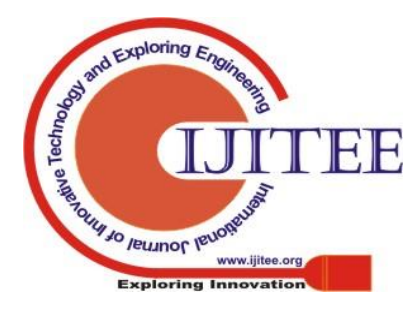




\section{Liver and Tumor Segmentation Techniques for CT Abdominal Images}

extracted is partitioned into three clusters in which one of the cluster is used to represent the background, the next cluster captures lesion pixels and the liver pixels are represented using other cluster. Post-processing is carried out by applying morphological operations like closing and opening which enhances the extracted lesions. The overall accuracy achieved for liver extraction is $96 \%$ and $97 \%$ is achieved for liver disease classification.

In [13], median filtering is used for preprocessing, and the segmentation is obtained by using binary tree quantization algorithm. The clustering algorithm is used for constructing binary tree and it is based on divide and conquer approach. Effective results that are better than K-means segmentation algorithm are achieved by using binary tree quantization algorithm.

In [14], computer aided diagnosis system is designed for liver cancer detection from the MRI, CT and US scan images. Segmentation of the liver image is done using $\mathrm{K}$-means clustering technique. Haar wavelet transform is applied for extracting the features which is used to calculate the threshold values of the region of interest. Next, thresholding is applied for tumors classification as cancer or non-cancerous. The experiment has generated an average accuracy of $82 \%$ which also reduces the complexity of time and computational complexity.

In [15], computer aided diagnostic system is designed for automatic extraction of liver and tumor. The liver is seperated using region growing method that start from an automatically detected seed point. Thresholding method is used for the analysis of intensity of the tumors and morphological erosion is applied to detect the tumors.

Table 1: Comparison of Literature survey

\begin{tabular}{|c|c|c|c|c|}
\hline Title & Techniques & Datasets & Limitations & Results \\
\hline 1. & $\begin{array}{l}\text { The liver is segmented by applying } \\
\text { region growing algorithm. }\end{array}$ & CT scan images & semi-automatic method & $\begin{array}{l}\text { Reliable and robust } \\
\text { liver segmentation. }\end{array}$ \\
\hline 2. & $\begin{array}{l}\text { Modified graph cuts for liver } \\
\text { segmentation }\end{array}$ & $\begin{array}{l}\text { Standard, } 20 \\
\text { 3Dircadb and } 20 \\
\text { Sliver07 } \\
\text { datasets }\end{array}$ & $\begin{array}{l}\text { segmentation of thin and } \\
\text { lengthy liver objects are } \\
\text { inaccurate and } \\
\text { Under-segmentation of } \\
\text { tumors. }\end{array}$ & $\begin{array}{l}\text { VOE, VD, VPT and } \\
\text { SSD is } 5.3 \%, 0.6 \% \text {, } \\
17.8 \mathrm{~s}, 1.0 \mathrm{~mm}: \\
\text { Sliver07 dataset and, } \\
8.6 \%, 0.7 \%, 12.7 \mathrm{~s}, \\
1.6 \mathrm{~mm}: 3 \text { Dircadb } \\
\text { dataset. }\end{array}$ \\
\hline 3. & MK-FCN for liver segmentation & $\begin{array}{l}400 \text { CT scanned } \\
\text { images }\end{array}$ & $\begin{array}{l}\text { The low-level layers of the } \\
\text { MK-FCN model are used to } \\
\text { extract features of tumors and } \\
\text { it is difficult to obtain deep } \\
\text { features. }\end{array}$ & $\begin{array}{l}\text { VOE: } 10.1 \%, \\
\text { RVD: } 5.2 \%, \\
\text { ASD: } 1.7 \%, \\
\text { RMSD: } 2.1 \mathrm{~mm} \text { and } \\
\text { MSD: } 6.1 \mathrm{~mm}\end{array}$ \\
\hline 4. & $\begin{array}{l}\text { FCM is used for liver segmentation. } \\
\text { DRLSE model to } \\
\text { extract features and detect tumors. }\end{array}$ & $\begin{array}{l}\text { Abdominal CT } \\
\text { scanned images }\end{array}$ & $\begin{array}{l}\text { Two images and over } \\
\text { segmentation }\end{array}$ & $\begin{array}{l}\text { first image - one } \\
\text { tumor extracted } \\
\text { Second image- four } \\
\text { tumors extracted. }\end{array}$ \\
\hline 5. & $\begin{array}{l}\text { Liver Segmentation: } \\
\text { 3D Mesh Deformation Model. } \\
\text { Tumor classification-SVM. }\end{array}$ & $\begin{array}{l}20 \text { CT scanned } \\
\text { images }\end{array}$ & Small dataset & $\begin{array}{l}\text { Liver segmentation }- \\
\text { an averaged Jaccard } \\
\text { Index }=0.93 \pm 0.05 . \\
\text { Liver tumor - an } \\
\text { average } \\
\mathrm{JI}=0.75 \pm 0.05 .\end{array}$ \\
\hline 6 & $\begin{array}{l}\text { Region growing - liver } \\
\text { segmentation, GLCM- feature } \\
\text { extraction, SVM-tumor } \\
\text { classification. }\end{array}$ & $\begin{array}{l}\text { Abdominal CT } \\
\text { scanned images } \\
\text { of } 70 \text { patients }\end{array}$ & $\begin{array}{l}\text { high computational } \\
\text { complexity }\end{array}$ & Accuracy $=82.3 \%$ \\
\hline 7 & $\begin{array}{l}\text { CLAHE-pre-processing, } \\
\text { Morphological operations-liver } \\
\text { segmentation, DWT-feature } \\
\text { extraction, } \\
\text { SVM- classification of HCC. }\end{array}$ & $\begin{array}{l}32 \text { patients } \\
\text { 3-D Abdominal } \\
\text { CT scanned } \\
\text { images }\end{array}$ & Less Accuracy & $\begin{array}{l}\text { Classification } \\
\text { accuracy } 88.46 \%\end{array}$ \\
\hline 8 & $\begin{array}{l}\text { Level set method with Gradient } \\
\text { Modification }\end{array}$ & $\begin{array}{l}\text { 3-D Abdominal } \\
\text { CT scanned } \\
\text { images }\end{array}$ & Dataset size is not mentioned & $\begin{array}{l}\text { Similarity coefficient } \\
\text { is higher than } 95 \% \text {. }\end{array}$ \\
\hline
\end{tabular}




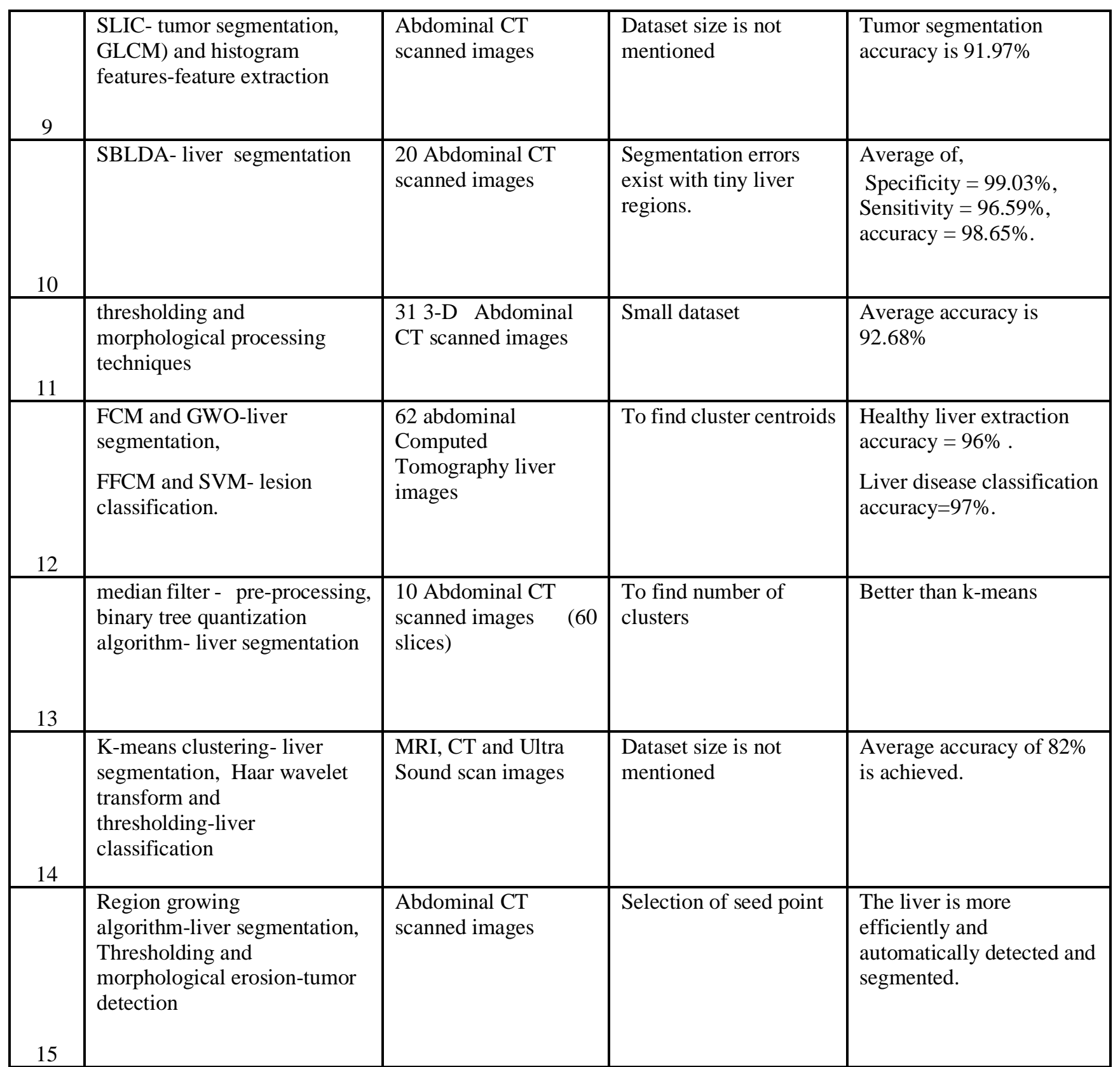

\section{IV.CONCLUSIONS}

The paper presents the elaborated discussion on already used techniques by various authors about segmentation of liver and in turn tumors from abdominal image of human body. This paper helps to understand the details about the work carried out and yet to be used techniques for further improvement in liver and tumor segmentation. Various new hybrid approaches can be evolved by using the ideas discussed in this paper.

\section{REFERENCES}

1. ZhengZhou, ZhengXueChang, Zheng Si ming "Semi- automatic Liver Segmentation in CT Images Through Intensity Separation and Region Growing", 8th International Congress of Information and Communication Technology, 2018.

2. Qing Huang, Hui Ding , Xiaodong Wang, Guangzhi Wang, "Fully automatic liver segmentation in CT images using modified graph cuts and feature detection”, Department of Biomedical Engineering, School of Medicine, Tsinghua University, Beijing 100084, 2018.

3. Changjian Sun1, Shuxu Guo1, "Liver Lesion Segmentation in CT Images with MKFCN", China, 2017.
4. P.Yugander, G. Raghotham Reddy, "Liver Tumor Segmentation in Noisy CT Images using Distance Regularized Level Set Evolution based on Fuzzy C-Means Clustering", 2nd IEEE International Conference On Recent Trends in Electronics Information \& Communication Technology (RTEICT), May 19-20, Chennai, 2017.

5. Jiayin Zhou, Member, IEEE, Yanling Chi, Member, IEEE, "Fast Segmentation and Modeling of Hepatic Components for the Planning of Robot-assisted Liver Tumor Ablation”, Singapore, IEEE, 2017.

6. Chao-Lun Kuo, Kuei-Fang Hsiao, "Texture-based Treatment Prediction by Automatic Liver Tumor Segmentation on Computed Tomography", Ministry of Science and Technology, IEEE, Taiwan, 2017.

7. Liaqat Ali1, Khaled Khelil, "Machine Learning Based Computer-Aided Diagnosis of Liver Tumours", IEEE International Conference On Cognitive Informatics and Cognitive Computing (ICCICC), 2017.

8. Wenqiang Cheng, Jiaqing Qiu, “A 3 - D Liver Tumor Level Set Method Based on Narrowband Gradient Modification”, IEEE, 2017.

9. Dr. M. V Sudhamani, Aravind H L, "Simple Linear Iterative Clustering based tumor Segmentation in Liver region of Abdominal CT-scans, IEEE, 2017.

10.Lianfen Huang, Minghui Weng, “Automatic Liver Segmentation from CT Images Using Single-Block Linear Detection”, Hindwai Publishing Corporation, China, 2016. 
11.L. Ali, A. Hussain, J. Li, A.A. Shah, U. Sudhakar "A Novel Fully Automated Liver and HCC Tumor Segmentation System using Morphological Operations", Research Gate, 2016.

12.Gehad Ismail Sayeda, Aboul Ella Hassaniena, Gerald Schaeferb, "An Automated Computer-Aided Diagnosis System for Abdominal CT Liver Images", International Conference on Medical Imaging Understanding and Analysis, MIUA, Loughborough, UK, Elsevier, 2016.

13.Vimtha.G.Sekhar, S N Kumar, Lenin fred, Sebastin Varghese, "An Improved Color Segmentation Algorithm for the Analysis Of Liver Anomalies in CT/PET Images", 2nd IEEE International Conference in Engineering and Technology (ICETECH), Coimbatore, TN, India 17 ${ }^{\text {th }}$ $\& 18^{\text {th }}$ March 2016.

14.Anisha P R, Kishor Kumar Reddy C, Narasimha Prasad L V, "A pragmatic approach for detecting liver cancer using image processing and data mining techniques", Dept of ECE, K L University, SPACES-2015.

15.M. Jayanthi, Dr. B.Kanmani, "Extracting the liver and tumor from abdominal CT images", $5^{\text {th }}$ International Conference on Signals and Image Processing, 2014.

\section{AUTHORS PROFILE}

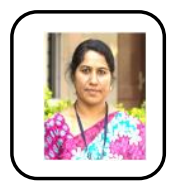

Mrs. Hema N, currently working as Assistant Professor, Dept. of ISE, RNSIT. She is having Teaching experience of 10 years and pursuing Ph.D in the area of Medical Image Processing.

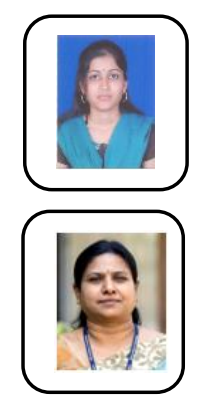

Mrs. Laxmidevi Noolvi, she is currently pursuing her $\mathrm{Ph} . \mathrm{D}$ degree in the department of Computer science and Engineering, RNSIT, Bangalore. She has carried out two research projects from VTU and AICTE. She has served as member of Board of Examiners (BOE) and Board of Study (BOS) member in VTU and other autonomous institutions across India. She has organized two international conferences ICDECS 2011 and 2015, and one more in December 2019. 